\title{
Downregulation of Long Non-Coding RNA AIRN Promotes Mitophagy in Alcoholic Fatty Hepatocytes by Promoting Ubiquitination of $\mathbf{m T O R}$
}

\author{
Sujian SHEN ${ }^{1}$, Jianzhang WANG ${ }^{1}$, Li miao LIN ${ }^{1}$ \\ ${ }^{1}$ Department of Gastroenterology, The Second Affiliated Hospital of Wenzhou Medical University, \\ Wenzhou, Zhejiang Province, China
}

Received July 29, 2020

Accepted January 28, 2021

Epub Ahead of Print March 8, 2021

\begin{abstract}
Summary
Long non-coding RNAs (IncRNAs) are crucial in chronic liver diseases, but the specific molecular mechanism of IncRNAs in alcoholic fatty liver (AFL) remains unclear. In this study, we investigated the in-depth regulatory mechanism of mTOR affected by AIRN non-protein coding RNA (IncRNA-AIRN) in the development of AFL. LncRNA-AIRN was highly expressed in the liver tissues of AFL C57BL/6 mice and oleic acid+alcohol $(O+A)$ treated AML-12 cells by using quantitative real-time PCR. RNA pull-down and RNA immunoprecipitation experiments demonstrated that there was an interaction between IncRNAAIRN and mTOR, and that interference with IncRNA-AIRN could promote the mTOR protein level. Results of cycloheximide-chase assay showed that the protein level of mTOR was decreased with the treatment time after the knockdown of IncRNA-AIRN. Furthermore, the knockdown of IncRNA-AIRN reduced mTOR protein level by promoting the E3 ubiquitin ligase FBXW7mediated ubiquitination. The IncRNA-AIRN/mTOR axis was involved in the regulation of the mitophagy of $\mathrm{O}+\mathrm{A}$ treated hepatocytes, which was confirmed by the cell transfection and the MTT assay. SPSS 16.0 was used for analyzing data. The difference between the two groups was analyzed by performing Student's t-test, and ANOVA was used to analyze the difference when more than two groups. $\mathrm{P}$ values $<0.05$ were considered to be significantly different. Our findings demonstrated that the knockdown of IncRNA-AIRN influenced mitophagy in AFL by promoting mTOR ubiquitination.
\end{abstract}

\section{Key words}

Alcoholic fatty liver • Mitophagy • IncRNA-AIRN • mTOR • Ubiquitination

\section{Corresponding author}

Li miao Lin, Department of Gastroenterology, The Second Affiliated Hospital of Wenzhou Medical University, Wenzhou, 325027, Zhejiang Province, China. E-mail: limiao_lin@126.com

\section{Introduction}

Alcoholic fatty liver (AFL) is a large accumulation of liver lipids as a result of alcohol consumption, which can further develop into local inflammation, focal necrosis, fibrosis, cirrhosis and even liver cancer (Seitz et al. 2018). In recent years, with the effective control of viral hepatitis type $B$, the incidence of AFL has been increasing year by year and alcohol is an important factor leading to the liver damage (Kong et al. 2019). According to the complicated pathogenesis of AFL, including mitochondrial $\beta$-oxidation, fatty acid oxidation, and the influence of immune factors and cytokines, but the specific pathogenesis is unclear (Stickel et al. 2017). As we all know, there is still a lack of effective treatment for AFL so far. Therefore, it is particularly necessary to elucidate the pathogenesis of AFL.

LncRNAs play a significant role in the process of transcription, post-transcription and protein translation, thus regulating the expressions of related molecules and widely affecting the occurrence of various diseases (Shi et al. 2013). A large number of studies have confirmed that lncRNAs are important in non-alcoholic fatty liver, liver fibrosis, and other chronic liver diseases. Even so, the specific role of IncRNAs in AFL is still not well 
understood (Li et al. 2019, Sulaiman et al. 2019). It was found that lncRNA-AIRN level was significantly up-regulated in high-fat diet (HFD)-fed mice, indicating that it was related to fatty liver disease (Guo et al. 2018, Oliva et al. 2009). However, the mechanism of IncRNAAIRN in AFL has not been resolved. In addition, the transport of mTOR to lysosomes in the liver cells of mice fed with ethanol increased the activation of mTOR, which results in the increase of mTOR activation (Chao et al. 2018). What's more, mTOR participates in the regulation of Parkin-mediated mitophagy under nutritional deficiency conditions (Jia et al. 2016).

Existing experiments have shown that autophagy, as an effective defense system against various complex pathological processes, can protect the liver from the alcohol damage by removing damaged mitochondria (Chao et al. 2018, Ye et al. 2019). Mitophagy is a unique form of autophagy, which can selectively remove damaged, folded, and redundant mitochondria by autophagolysosomes, so as to maintain the balance between the quality and quantity of mitochondria and cell survival (Ge et al. 2019). Although a large number of studies have confirmed that promoting mitophagy through the PINK1/Parkin signaling pathway contributes to alleviating AFL, the specific regulatory mechanism is not yet clear (Hao et al. 2019).

In this study, we predicted that there was a binding site between IncRNA-AIRN and mTOR through the RPISeq software. Furthermore, we aimed to investigate the in-depth regulatory mechanism of mTOR affected by lncRNA-AIRN in the development of AFL.

\section{Methods}

\section{Experimental animals}

A total of 12 male C57BL/6 mice, 8 weeks old, were purchased from Shanghai Experimental Animal Research Center, and were housed in a standard animal housing facility and provided with food and water at discretion. To establish the mouse model of AFL, the mice were administered on the basis of feeding with a Lieber-DeCarli high-protein liquid diet: one containing $36 \%$ ethanol (ETOH)-derived calories (AFL group) or one made isocaloric with dextrin and maltose with a pairfeeding protocol (Sham group). Our study was approved by the Institute's Research Ethics Committee of Wenzhou Medical University. All animals care and experiment protocols were performed strictly according the Principles of Laboratory Animal Care.

\section{Cell culture}

The normal hepatocytes AML-12 were purchased from the Cell Bank of Chinese Academy of Science (Shanghai, China). AML-12 cells were grown in the DMEM: F12 culture medium $(500 \mathrm{ml})$, which contains $10 \%$ fetal bovine serum (FBS), $10 \mu \mathrm{g} / \mathrm{ml}$ insulin, $5.5 \mu \mathrm{g} / \mathrm{ml}$ transferrin, $5 \mathrm{ng} / \mathrm{ml}$ selenium, $40 \mathrm{ng} / \mathrm{ml}$ dexamethasone, and cultured at $37{ }^{\circ} \mathrm{C}$ in an environment of $5 \% \mathrm{CO}_{2}$ atmosphere.

\section{Cell transfection}

AML-12 cells were plated in a $24-$ well plate at $1 \times 10^{5}$ per well until the confluence reached $70 \%$. The small interfering RNAs (siRNAs) were designated as si-AIRN, and the full-length mTOR cDNA was PCR amplified and cloned into the pcDNA3.1 vector (pcDNAmTOR). Then, si-AIRN and pcDNA-mTOR were transfected into the cells using Lipofectamine 2000 (Invitrogen). Then, the efficiency of transfection was analyzed by quantitative real-time PCR (qRT-PCR). The empty pcDNA3.1 vector and si-control were utilized as negative controls.

Quantitative real-time polymerase chain reaction ( $q R T-P C R)$

Total RNAs in the study were extracted from cultured cells by the use of TRIzol reagent (Thermo Fisher Scientific, USA) according to the manufacturer's instructions. The SYBR Green PCR Kit (Takara) on the ABI 7500 Fast Real-time PCR system (Applied Biosystems, USA) was used for conducting the qRT-PCR. GAPDH was used as an internal reference. The $2^{-\Delta \Delta \mathrm{Ct}}$ method was performed to analyze the relative expression levels after normalized.

\section{Enzyme-linked immunosorbent assay (ELISA)}

From the modeling to the fourth weekend, blood was drawn from the angular vein of the eyes after anesthesia. The serum was separated and collected to analyze the serum levels of alanine aminotransferase (ALT) and aspartate aminotransferase (AST). The concentrations of ALT and AST were measured by ELISA kits in accordance with the manufacturer's instructions.

\section{Extracellular triglycerides and total cholesterol assays}

Enzyme coupled colorimetric method was carried out for measurement of serum triglycerides (TG) and total cholesterol (TC). 


\section{Western blot}

The expressions of genes at protein levels were evaluated by western blot analysis. Cells used in this study were lysed on ice for 30 min followed by using RIPA (Radio Immunoprecipitation Assay) lysis buffer with protease inhibitors. Protein concentrations in the cell lysates were determined using the Bradford assay (BioRad, Philadelphia, PA, USA). Proteins were separated through SDS-PAGE, and transferred onto PVDF membranes subsequently. Following being blocked by $5 \%$ skimmed milk powder for $1 \mathrm{~h}$ at room temperature, the PVDF membranes were incubated at $4{ }^{\circ} \mathrm{C}$ overnight with primary antibody and then washed with TBST. Thereafter, they were immunoblotted with the secondary antibody for $2 \mathrm{~h}$, and then detected by Enhanced Chemiluminescent (ECL) detection reagents (Thermo Scientific, Shanghai, China). The primary antibodies used in this study were specifically against mTOR, LC3II/LC3I, PINK1 and Parkin (Abcam). Antibody $\beta$-actin (1:1000, ab8227, Abcam) was used as the control.

\section{MTT assay}

MTT assay was used to measure the viability of the cells. After transfection, cells were seeded in a 96-well plate at a density of $1 \times 10^{3}$, and cultured for the next $24 \mathrm{~h}$. MTT powder was dissolved in PBS as a stock solution. Then, the MTT solution was added to each well and incubated for $4 \mathrm{~h}$ at $37{ }^{\circ} \mathrm{C}$. Thereafter, dimethyl sulfoxide (DMSO) was added to each well and the plate was shaken gently. Finally, the optical density (OD) value at $570 \mathrm{~nm}$ was measured for each well.

\section{RNA pull-down assay}

An RNA pull-down assay was performed using the Pierce Magnetic RNA-Protein Pull-Down Kit (Thermo Fisher, CA). In short, after the biotinylated lncRNA-AIRN was transfected into AML-12 cells, the cells were lysed with lysis buffer at room temperature and incubated with biotinylated probes. The cell lysates were then mixed with streptavidin agarose beads (Sigma) overnight. Finally, the RNA-protein mixture was boiled in the SDS buffer for $10 \mathrm{~min}$, and mTOR protein level in the IncRNA-AIRN pull-down complex was analyzed by western blotting.

\section{RNA immunoprecipitation (RIP)}

The RIP assay was performed by the use of a Millipore EZ-Magna RIP RNA-Binding Protein Immunoprecipitation kit (Millipore, MA, USA). Briefly, the obtained AML-12 cells were incubated in RIP buffer for $30 \mathrm{~min}$. Then they were incubated with magnetic beads conjugated to the mTOR antibody (Millipore, USA) for $6 \mathrm{~h}$ at $4{ }^{\circ} \mathrm{C}$. Finally, the level of lncRNA-AIRN in the coprecipitation was detected through qRT-PCR.

\section{Cycloheximide treatment and ubiquitination assay}

Cycloheximide (CHX) is a protein synthesis inhibitor that can investigate the effect of lncRNA-AIRN on mTOR degradation. After AML-12 cells were transfected with si-AIRN, each group of cells was treated with $50 \mu \mathrm{g} / \mathrm{ml} \mathrm{CHX}$. The mTOR protein level was detected at $0,3,6$, and $9 \mathrm{~h}$, respectively.

In the ubiquitination assay, AML-12 cells were transfected with HA-tagged $\mathrm{Ub}$ (HA-Ub) and Flag-tagged mTOR (Flag-mTOR), and then treated with the protease inhibitor MG132 (10 $\mu \mathrm{M})$. Next, si-AIRN was transfected into the cells to downregulate mTOR. Forty-eight hours later, whole-cell lysates were prepared using a cell lysis buffer and immunoprecipitated using anti-mTOR antibodies, and then incubated with immunomagnetic beads overnight. Finally, the complex was analyzed through western blot to detect ubiquitination by using anti-Ub antibodies.

\section{Co-Immunoprecipitation (CO-IP)}

CO-IP is applied to examine the potential protein interaction between FBXW7 and mTOR. After being transfected with si-AIRN, the AML-12 cells were incubated with the flag lysis buffer with freshly prepared protease inhibitors. Then, cell lysates were incubated with antibodies (mTOR or FBXW7) or control normal IgG for $3 \mathrm{~h}$ and $30 \mu \mathrm{l}$ of protein $\mathrm{A} / \mathrm{G}$ immunoprecipitation magnetic beads (B23202, Biotool, USA) for $12 \mathrm{~h}$ at $4{ }^{\circ} \mathrm{C}$. The resulting immune-complexes were subjected to SDSPAGE and detected by western blot using antibody FBXW7 or mTOR.

\section{Statistics analysis}

SPSS 16.0 (SPSS Inc, Chicago, USA) was used for analyzing data that collected from at least three independent experiments. All results were represented as mean \pm standard deviation (SD). The difference between the two groups was analyzed by performing Student's t-test, and ANOVA was used to analyze the difference when more than two groups. $\mathrm{P}$ values $<0.05$ were considered to be significantly different. 


\section{Results}

Up-regulation of IncRNA-AIRN expression in liver tissues and cells of AFL mice

To simulate the model of humans with AFL, C57BL/6 mice were treated with alcohol in drinking water. Compared with Sham group, the levels of ALT, AST and TG in the AFL group were significantly higher

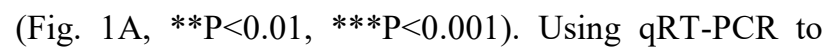
detect the expression of IncRNA-AIRN in mice liver tissues, we found that the expression level of IncRNAAIRN in the AFL group was higher than that in the Sham group (Fig. 1B, $* * * \mathrm{P}<0.001$ ). Next, we detected the expression of mTOR and mitophagy-related proteins LC3II/LC3I, PINK1 and Parkin. From the results of western blot, it was found that compared with Sham group, mTOR was significantly highly expressed in AFL group, while the protein levels of LC3II/LC3I, PINK1, and Parkin were down-regulated (Fig. 1C).

In addition, we treated immortalized normal hepatocytes (AML-12) with $100 \mu \mathrm{mol} / \mathrm{l}$ oleic acid and $87 \mu \mathrm{mol} / \mathrm{l}$ ethanol $(\mathrm{O}+\mathrm{A})$ for $48 \mathrm{~h}$ to establish an in vitro model (Tang et al. 2016). The qRT-PCR and western blot assays indicated that the levels of IncRNA-AIRN and mTOR in cells were upregulated in the $\mathrm{O}+\mathrm{A}$ group compared with the normal hepatocytes, while LC3II/LC3I, PINK1, and Parkin were downregulated (Fig. 1D,E, ${ }^{* * P}<0.01$ ). Also, we examined the levels of TC and TG in cultured cells and found that TC and TG were significantly higher in the $\mathrm{O}+\mathrm{A}$ group (Fig. $1 \mathrm{~F}$, **P $<0.01)$. Through the MTT assay we found that the AFL cell viability was relatively low compared to controls (Fig. 1G, $* * \mathrm{P}<0.01$ ). Based on the above experimental results, we inferred that lncRNA-AIRN was highly expressed in the liver tissues and hepatocytes of AFL mice.
$\mathbf{A}$

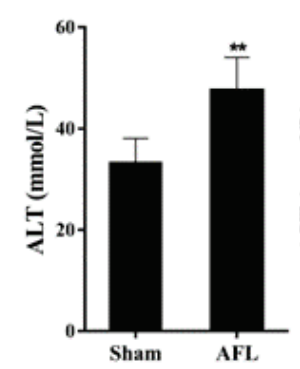

D

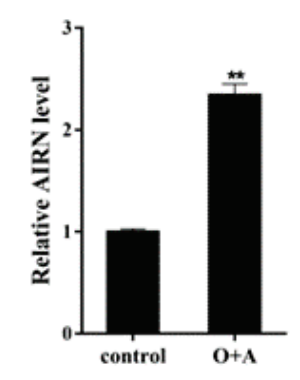

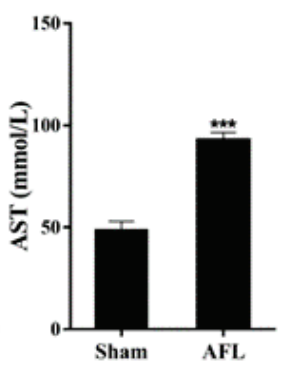

$\mathbf{E}$

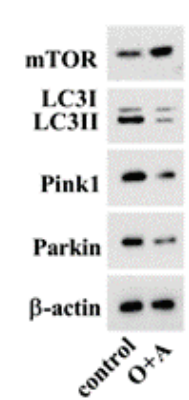

B

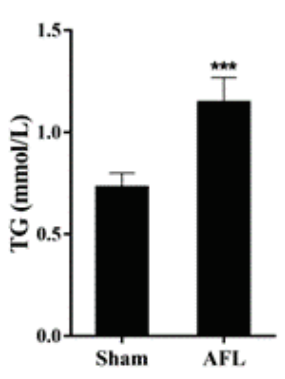

F

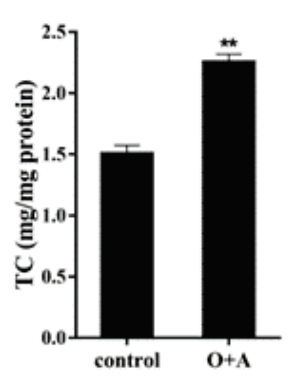

C

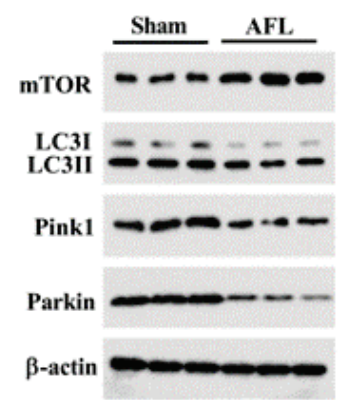

G
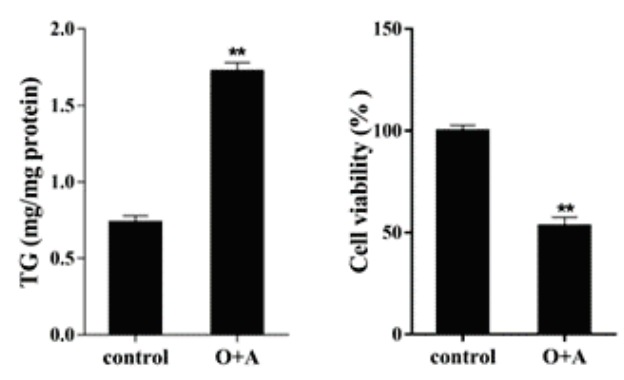

Fig. 1. Upregulation of IncRNA-AIRN expression in AFL liver tissues and alcohol-induced cells. The Eight-week-old C57BL/6 mice ( $n=12$ ) were randomly divided into 2 groups, namely Sham group and AFL group. (A) The levels of alanine aminotransferase (ALT), aspartate aminotransferase (AST) and triglycerides (TG) in serum were detected by ELISA. (B) LncRNA-AIRN level was detected using qRT-PCR. (C) The expressions of mTOR, LC3II/LC3I, PINK1, and Parkin were detected by western blot. $* * P<0.01, * * * P<0.001$ vs Sham. AML-12 cells were treated with $100 \mu \mathrm{mol} / \mathrm{L}$ oleic acid and $87 \mu \mathrm{mol} / \mathrm{L}$ ethanol $(\mathrm{O}+\mathrm{A})$ to establish an in vitro model, which was divided into control group and O+A group. (D) LncRNA-AIRN level was measured by qRT-PCR. (E) The expressions of mTOR, LC3II/LC3I, PINK1 and Parkin were also detected by western blot. (F) The levels of TC and TG were examined by enzyme coupled colorimetric method. (G) The MTT assay was used to detect the cell viability. ${ }^{* * P}<0.01$ vs control.

Interfering with lncRNA-AIRN promotes hepatocyte mitophagy

To determine whether interfering lncRNA-AIRN can affect hepatocyte mitophagy, AML-12 cells were transfected with si-AIRN and si-control, and then treated with $100 \mu \mathrm{mol} / \mathrm{l}$ oleic acid and $87 \mu \mathrm{mol} / \mathrm{l}$ ethanol $(\mathrm{O}+\mathrm{A})$ for $48 \mathrm{~h}$. In the $\mathrm{O}+\mathrm{A}+\mathrm{si}-\mathrm{AIRN}$ group, the levels of IncRNA-AIRN (Fig. 2A, *** $<<0.001$ ) and mTOR 
(Fig. 2B) were remarkably downregulated. On the country, the protein levels of LC3II/LC3I, PINK1, and Parkin were upregulated and the levels of TC and TG were higher (Fig. 2C, $* * \mathrm{P}<0.01, * * * \mathrm{P}<0.001$ ), whereas the cell viability (Fig. $2 \mathrm{D},{ }^{*} \mathrm{P}<0.05$ ) was higher compared with the other two groups. It could be concluded from the above experimental results that the interference with lncRNA-AIRN in hepatocytes could promote mitophagy.

\section{The interaction between IncRNA-AIRN and mTOR}

RPISeq predicted that there was an interaction between IncRNA-AIRN and mTOR. To further investigate the correlation between IncRNA-AIRN and mTOR, an RNA pull-down experiment was performed to detect the protein expression of mTOR in the IncRNAAIRN pull-down complex (Hosen et al. 2018) (Fig. 3A). In parallel with our hypothesis, the level of lncRNAAIRN was high in protein samples precipitated by mTOR antibodies using RIP assay and qRT-PCR (Fig. 3B, $* \mathrm{P}<0.05)$. Moreover, the results of the $\mathrm{qRT}-\mathrm{PCR}$ and western blot showed that interfering with lncRNA-AIRN in the AML-12 cells significantly inhibited the expression of mTOR protein (Fig. 3C, $* * * \mathrm{P}<0.001$ ). However, there was not marked change in the mRNA level of mTOR. Together, these data demonstrated that there was an interaction between IncRNA-AIRN and mTOR, and that interference with IncRNA-AIRN could suppress the expression of the mTOR protein.

Effect of the interference with IncRNA-AIRN on ubiquitination of $m$ TOR

Given the unchanged mTOR mRNA level and the changed protein level affected by $\operatorname{lncRNA}-A I R N$, we inferred that lncRNA-AIRN might affect the mTOR level by mediating the ubiquitination degradation of mTOR. After being transfected with si-AIRN, the AML-12 cells
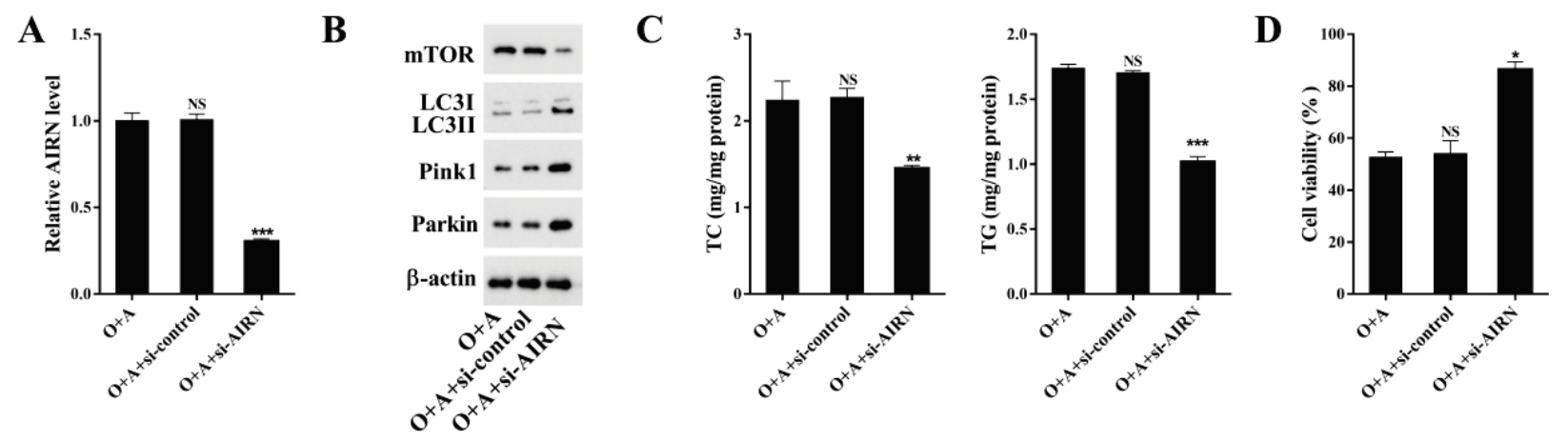

Fig. 2. Interfering with IncRNA-AIRN promotes hepatocyte mitophagy. AML-12 cells were transfected with si-AIRN and si-control and treated with $100 \mu \mathrm{mol} / \mathrm{l}$ oleic acid and $87 \mu \mathrm{mol} / \mathrm{l}$ ethanol, which was divided into O+A, O+A+si-control and O+A+si-AIRN group. (A) LncRNA-AIRN level was measured by qRT-PCR. (B) The expressions of mTOR, LC3II/LC3I, PINK1 and Parkin were also detected by western blot. (C) The levels of TC and TG were examined by enzyme coupled colorimetric method. (D) The MTT assay was used detect the cell viability. $* \mathrm{P}<0.05,{ }^{*} \mathrm{P}<0.01, * * * \mathrm{P}<0.001$ vs $\mathrm{O}+\mathrm{A}+$ si-control.

A

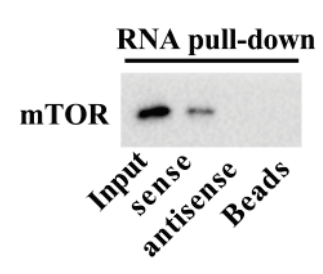

B

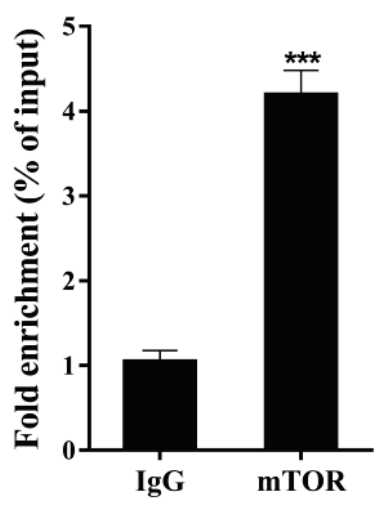

C

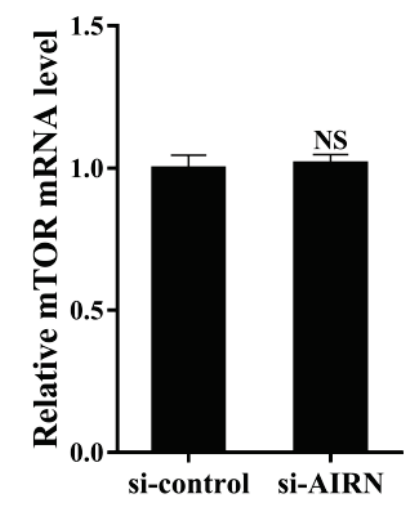

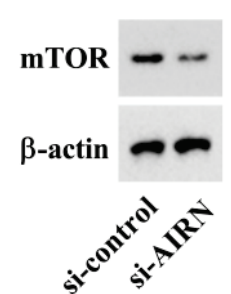

Fig. 3. The interaction between IncRNA-AIRN and mTOR. (A, B) An RNA pull-down and RIP assays were carried out to detect the interaction between mTOR and IncRNA-AIRN. (C) The expression of mTOR was estimated by performing qRT-PCR and western blot. $* * * \mathrm{P}<0.001$ vs IgG. 
were treated with $\mathrm{CHX}$ for $0,3,6$, and $9 \mathrm{~h}$, respectively. The results showed that in the si-AIRN group, the protein level of mTOR was gradually decreased with the prolonged $\mathrm{CHX}$ treatment time, while the expression of mTOR in the control group did not change significantly (Fig. 4A). To further explore the effect of lncRNA-AIRN on mTOR protein, the cells with transfected IncRNAAIRN were treated with $10 \mu \mathrm{mol} / 1$ proteasome inhibitor MG132 for $4 \mathrm{~h}$ (Kim et al. 2020). As shown in the Fig. 4B, the protein level of mTOR was significantly increased in the si-AIRN group with MG132 treatment, which was barely changed in the si-control group. Based on this, we indicated that proteasome-mediated mTOR protein degradation was successfully blocked (Fig. 4B, $\# \# \mathrm{P}<0.01, * * * \mathrm{P}<0.001)$. Furthermore, the ubiquitination and Co-IP assays revealed that the interference with IncRNA-AIRN enhanced the ubiquitination of mTOR and the interaction between $\mathrm{mTOR}$ and E3 ubiquitin ligase FBXW7 (Fig. 4C,D, **P<0.01). Therefore, the outcomes confirmed that interfering with lncRNA-AIRN accelerated mTOR degradation and promoted ubiquitination mediated by E3 ubiquitin ligase FBXW7.
A

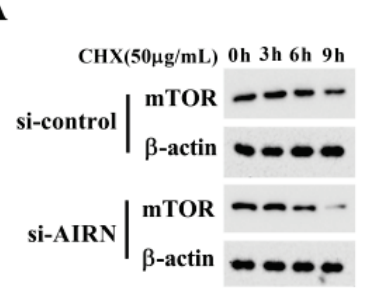

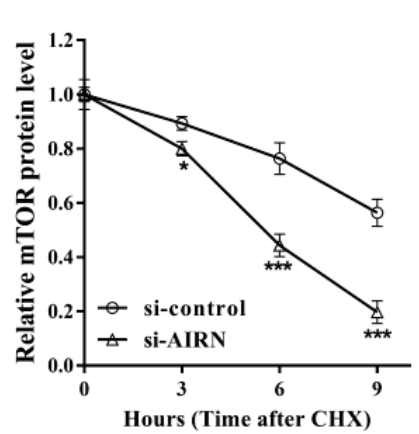

$\mathbf{D}$
B

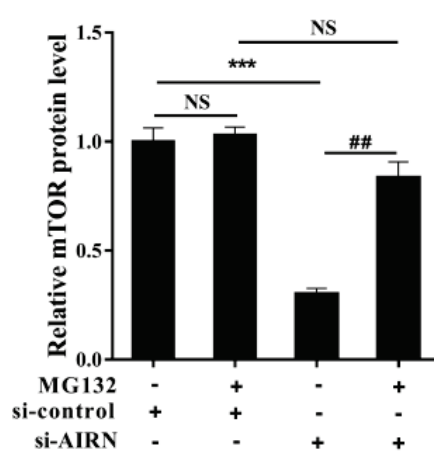

C

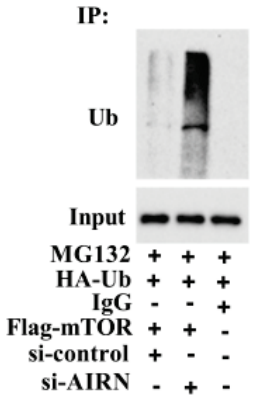

IB:

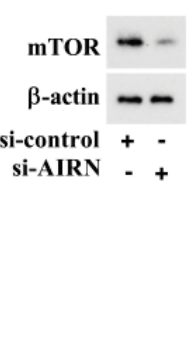

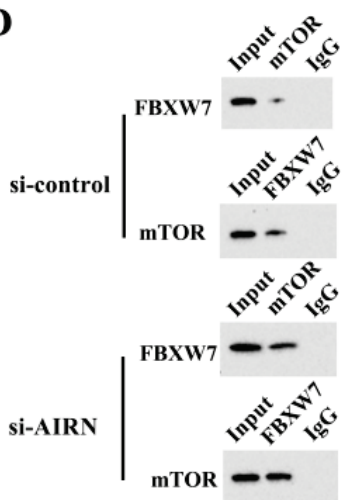
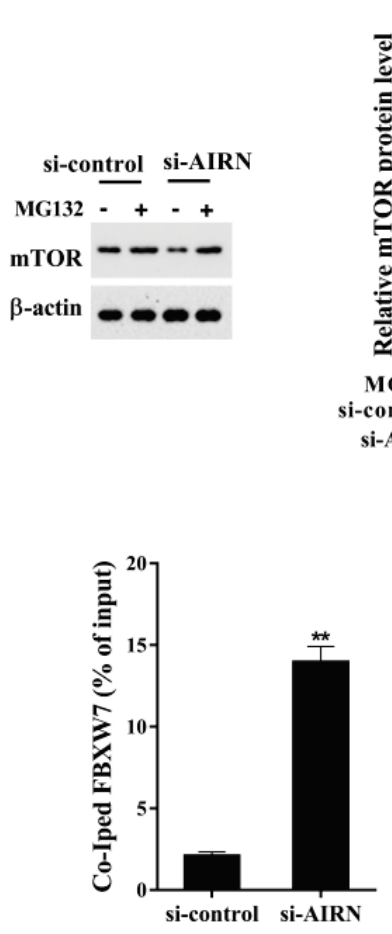

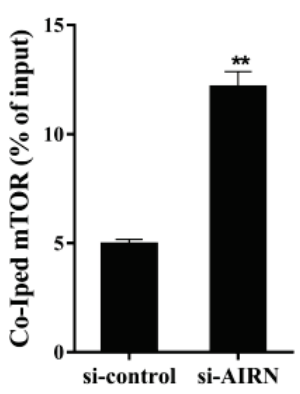

Fig. 4. Effect of the interference with IncRNA-AIRN on ubiquitination of mTOR. (A) The relative mTOR expression in AML-12 cells at 0 , 3, 6, and $9 \mathrm{~h}$ after being co-treated with si-AIRN and $\mathrm{CHX}(50 \mu \mathrm{g} / \mathrm{ml})$. (B) The AML-12 cells with transfected si-AIRN were treated with $10 \mu \mathrm{M}$ proteasome inhibitor MG132. mTOR expression was examined by Western blot. (C) Ubiquitination of mTOR was increased after interfering IncRNA-AIRN. (D) The binding of mTOR to FBXW7 was validated via conducting CO-IP assay. $* P<0.05, * * P<0.01$, $* * * P<0.001$ vs si-control, \#\#P<0.01 vs si-AIRN.

Interfering with lncRNA-AIRN promotes mitophagy via downregulating mTOR

To explore the effect of lncRNA-AIRN/mTOR pathway in AFL, the AML-12 cells were transfected with si-AIRN, and the rescue experiment was performed by the co-transfection with si-AIRN and pcDNA-mTOR. Then, we used $100 \mu \mathrm{mol} / 1$ oleic acid and $87 \mu \mathrm{mol} / \mathrm{l}$ ethanol $(\mathrm{O}+\mathrm{A})$ for $48 \mathrm{~h}$ to establish an in vitro model. The results showed that the protein level of mTOR was increased by the co-transfection with si-AIRN and
pcDNA-mTOR (Fig. 5A). In addition, the levels of TC and $\mathrm{TG}$ in the $\mathrm{O}+\mathrm{A}+\mathrm{si}-\mathrm{AIRN}+$ pcDNA-mTOR group were higher than $\mathrm{O}+\mathrm{A}+$ si-AIRN group (Fig. 5B, $* \mathrm{P}<0.05, \quad * * \mathrm{P}<0.01)$. The cell viability of $\mathrm{O}+\mathrm{A}+$ si-AIRN+pcDNA-mTOR group was relatively low compared to $\mathrm{O}+\mathrm{A}+$ si-AIRN group (Fig. 5C, $* \mathrm{P}<0.05$ ). Taken together, our findings demonstrated that interfering with lncRNA-AIRN promotes mitophagy via reducing mTOR. 
A

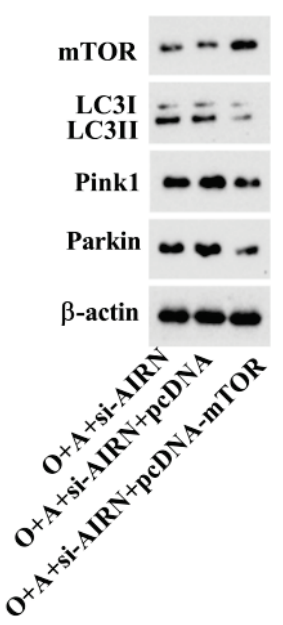

B

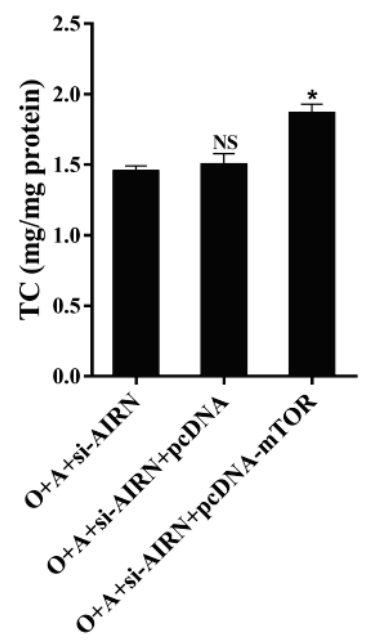

C

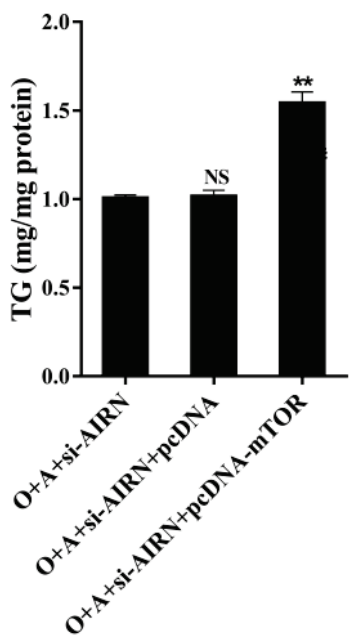

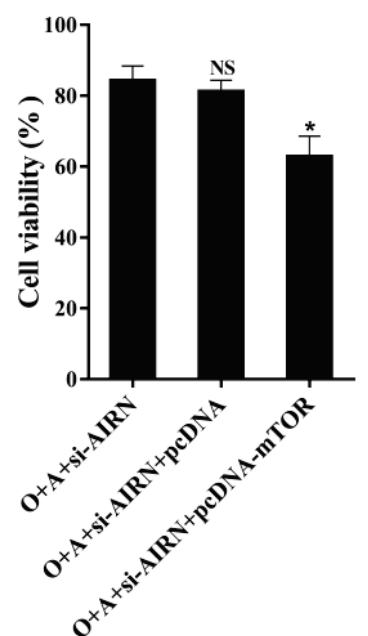

Fig. 5. Interfering with IncRNA-AIRN promotes mitophagy via downregulating mTOR. The experiment was performed by the co-transfection with si-AIRN and pcDNA-mTOR. (A) The expressions of mTOR, LC3II/LC3I, PINK1 and Parkin were also detected by western blot. (B) The levels of TC and TG were examined by enzyme coupled colorimetric method. (C) The MTT assay was used detect the cell viability. ${ }^{*} \mathrm{P}<0.05,{ }^{*} \mathrm{P}<0.01$ vs $\mathrm{O}+\mathrm{A}+\mathrm{si}-\mathrm{AIRN}+\mathrm{pcDNA}$.

\section{Discussion}

In the current study, we provide the first experimental evidence of a crucial role of lncRNA-AIRN in improving AFL via inhibiting ubiquitination degradation of mTOR. The present research demonstrated that IncRNA-AIRN and mTOR were both upregulated in AFL liver tissues and $\mathrm{O}+\mathrm{A}$-induced cells. Furthermore, we investigated that interference with IncRNA-AIRN promoted mitophagy by interacting with $\mathrm{mTOR}$ to promote its ubiquitination degradation.

As we know, IncRNA refers to a type of RNA with a transcript length of more than 200 nucleotides, which is lack of protein coding capacity (Li et al. 2014). LncRNA-AIRN has been proven to be associated with fatty liver disease, and the level of lncRNA-AIRN was significantly upregulated that occurred in response to a treatment of high-fat diet (HFD) (Guo et al. 2018). Consistent with the previous studies, we also found that IncRNA-AIRN was abnormally highly expressed in AFL liver tissues and $\mathrm{O}+\mathrm{A}$-induced cells. What's more, PINK1/Parkin is considered to be a main way to mediate mitophagy, in which the change of PINK1 protein level can directly reflect the level of mitophagy in cells (Eid et al. 2016). Notably, we observed the protein level of PINK1 and Parkin was upregulated after interfering with lncRNA-AIRN in hepatocytes. These findings strongly indicated that IncRNA-AIRN had an important influence on mitophagy, which further affects AFL. Although the effects of IncRNA AIRN on mitophagy were explored, its effects on apoptosis and necrosis of hepatocytes were not studied. In addition, we did not further analyze the relationship between IncRNA AIRN/mTOR signal pathway and alcoholic fatty liver disease using clinical data of healthy subjects and patients with alcoholic fatty liver disease. Therefore, further research is needed to address these limitations.

Currently, there are studies elucidating that mTOR participates in the regulation of Parkin-mediated mitophagy. In this study, we found that there was a binding relationship between IncRNA-AIRN and mTOR and that interference with IncRNA-AIRN could inhibit the expression of the mTOR protein. However, it remains unknown how mTOR exerts functions in the process of mitophagy. Therefore, the mechanism of mTOR in regulating mitophagy was explored in our study. Given that the unchanged mTOR mRNA levels and the significantly changed protein levels affected by IncRNA-AIRN, we inferred that IncRNA-AIRN might affect the mTOR level by mediating the ubiquitination degradation. Ubiquitination is a post-translational protein modification that can regulate extracellular signals by mediating protein activity or protein degradation (Malynn and $\mathrm{Ma}$ 2010). The three-step cascade of ubiquitin activation, coupling and transfer is co-mediated by E1 ubiquitin kinase, E2 ubiquitin binding enzyme and E3 ubiquitin ligase. Given that E3 ubiquitin ligase can specifically recognize the substrate, it is considered to be the most important molecule in the ubiquitination (Malynn and Ma 2010). Herein, our findings indicated 
that E3 ubiquitin ligase FBXW7 promotes mTOR ubiquitination degradation by binding to mTOR, which which is consistent with previous studies (Xu et al. 2016). However, we did not further study the effect of IncRNA AIRN on the downstream pathway of mTOR, and the regulatory mechanism will be further studied in the future.

On the whole, the present study demonstrated that IncRNA-AIRN may inhibit mitophagy and promote alcohol-induced hepatocyte damage via upregulating mTOR, while lncRNA-AIRN silencing activated the
PINK1/Parkin signaling pathway to promote mitophagy and reduce lipid accumulation, suggesting a potential road for lncRNA-AIRN in treating AFL.

\section{Conflict of Interest}

There is no conflict of interest.

\section{Acknowledgements}

The datasets used and/or analyzed during the current study are available from the corresponding author on reasonable request.

\section{References}

CHAO XJ, WANG SG, ZHAO K, LI Y, WILLIAMS JA, LI TG, CHAVAN H, KRISHNAMURTHY P, HE XC, LI LH, BALLABIO A, NI HM, DING WX: Impaired TFEB-mediated lysosome biogenesis and autophagy promote chronic ethanol-induced liver injury and steatosis in mice. Gastroenterology 155: 865-879, 2018. https://doi.org/10.1053/j.gastro.2018.05.027

EID N, ITO Y, OTSUKI Y: Triggering of Parkin mitochondria! Translocation in mitophagy: Implications for liver diseases. Front Pharmacol: 100, 2016. https://doi.org/10.3389/fphar.2016.00100

GE B, WU H, SHAO D, LI S, LI F: Interfering with miR-24 alleviates rotenone-induced dopaminergic neuron injury via enhancing autophagy by up-regulating DJ-1. Aging Pathobiol Ther 1: 17-24, 2019. https://doi.org/10.31491/APT.2019.12.004

GUO J, ZHOU Y, CHENG Y, FANG W, HU G, WEI J, LIN Y, MAN Y, GUO L, SUN M, CUI Q, LI J: Metformininduced changes of the coding transcriptome and non-coding RNAs in the livers of non-alcoholic fatty liver disease mice. Cell Physiol Biochem 45: 1487-1505, 2018. https://doi.org/10.1159/000487575

GAO H, LV Y, LIU Y, LI J, WANG X, ZHOU Z, TIPOE GL, OUYANG S, GUO Y, ZHANG J, HAO X, LI W, KOIKE K, SO KF, XIAO J: Wolfberry-derived zeaxanthin dipalmitate attenuates ethanol-induced hepatic damage. Mol Nutr Food Res: 63:e1801339. https://doi.org/10.1002/mnfr.201801339

HOSEN MR, MILITELLO G, WEIRICK T, PONOMAREVA Y, DASSANAYAKA S, MOORE JB 4TH, DÖRING C, WYSOCZYNSKI M, JONES SP, DIMMELER S, UCHIDA S: Airn regulates Igf2bp2 translation in cardiomyocytes. Circ Res 122: 1347-1353, 2018. https://doi.org/10.1161/CIRCRESAHA.117.312215

JIA SN, LIN C, CHEN DF, LI AQ, DAI L, ZHANG L, ZHAO LL, YANG JS, YANG F, YANG WJ: The transcription factor $\mathrm{p} 8$ regulates autophagy in response to palmitic acid stress via a mammalian target of rapamycin (mTOR)-independent signaling pathway. J Biol Chem 291: 4462-4472, 2016. https://doi.org/10.1074/jbc.M115.675793

KIM SY, KIM HJ, KIM HJ, KIM CH: Non-thermal plasma induces antileukemic effect through mTOR ubiquitination. Cells 9:pii: E595, 2020. https://doi.org/10.3390/cells9030595

KONG LZ, CHANDIMALI N, HAN YH, LEE DH, KIM JS, KIM SU, KIM TD, JEONG DK, SUN HN, LEE DS, KWON T: Pathogenesis, early diagnosis, and therapeutic management of alcoholic liver disease. Int J Mol Sci 20: 2712, 2019. https://doi.org/10.3390/ijms20112712

LI HD, DU XS, HUANG HM, CHEN X, YANG Y, HUANG C, MENG XM, LI J: Noncoding RNAs in alcoholic liver disease. J Cell Physiol 234: 14709-14720, 2019. https://doi.org/10.1002/jcp.28229

LI X, WU Z, FU X, HAN W: lncRNAs: Insights into their function and mechanics in underlying disorders. Mutation Res 762: 1-21, 2014. https://doi.org/10.1016/j.mrrev.2014.04.002

MALYNN BA, MA A: Ubiquitin makes its mark on immune regulation. Immunity 33: 843-852, 2010. https://doi.org/10.1016/j.immuni.2010.12.007

OLIVA J, BARDAG-GORCE F, FRENCH BA, LI J, FRENCH SW: The regulation of non-coding RNA expression in the liver of mice fed DDC. Exp Mol Pathol 87: 12-19, 2009. https://doi.org/10.1016/j.yexmp.2009.03.006 
SEITZ HK, BATALLER R, CORTEZ-PINTO H, GAO B, GUAL A, LACKNER C, MATHURIN P, MUELLER S, SZABO G, TSUKAMOTO H: Alcoholic liver disease. Nat Rev Dis Primers 16, 2018. HTTPS://DOI.ORG/10.1038/S41572-018-0014-7

SHI X, SUN M, LIU H, YAO Y, SONG Y: Long non-coding RNAs: a new frontier in the study of human diseases. J Thorac Oncol 339: 159-166, 2013. https://doi.org/10.1016/j.canlet.2013.06.013

STICKEL F, DATZ C, HAMPE J, BATALLER R: Pathophysiology and management of alcoholic liver disease. Gut Liver. 447-447, 2017. https://doi.org/10.5009/gnl11031

SULAIMAN SA, MUHSIN NIA, JAMAL R: Regulatory non-coding RNAs network in non-alcoholic fatty liver disease. Front Physiol 10: 279, 2019. https://doi.org/10.3389/fphys.2019.00279

TANG LY, CHEN Y, RUI BB, HU CM: Resveratrol ameliorates lipid accumulation in HepG2 cells, associated with down-regulation of lipin1 expression. Can J Physiol Pharm 94: 185-189, 2016. https://doi.org/10.1139/cjpp2015-0125

XU Y, TIAN C, SUN J, ZHANG J, REN K, FAN XY, WANG K, WANG H, YAN YE, CHEN C, SHI Q, DONG XP: FBXW7-induced mTOR degradation forces autophagy to counteract persistent prion infection. Mol Neurobiol 53: 706-719, 2016. https://doi.org/10.1007/s12035-014-9028-7

YE X, ZHOU XJ, ZHANG H: Autophagy in immune-related renal disease. J Immunol Res 2019: 5071687, 2019. https://doi.org/10.1155/2019/5071687 